\title{
Prognostic Significance of Lymph Node Ratio in Stage III Rectal Cancer
}

\author{
Jin Yong Shin, Kwan Hee Hong ${ }^{1}$ \\ Department of Surgery, Haeundae Paik Hospital, ${ }^{1}$ Pusan Paik Hospital, Inje University College of Medicine, Busan, Korea
}

Purpose: Although nodal metastasis is the most powerful prognostic factor in rectal cancer, marked heterogeneity exists within stage III rectal cancer. Recent studies of rectal cancer have shown a prognostic superiority of the lymph node ratio (LNR) compared with $\mathrm{N}$ stage. The purpose of this study was to investigate the prognostic value of the LNR in the era of the 7th edition of the TNM classification.

Methods: We included 190 patients who underwent a curative resection for rectal cancer with nodal metastasis. The patients were divided into four groups on the basis of statistically calculated cut-off values as $0.21,0.32$, and 0.61 .

Results: The LNR was an independent risk factor for overall survival (OS; $\mathrm{P}=0.008)$ and for systemic recurrence-free survival (SRFS; $\mathrm{P}=0.002$ ). However, the LNR was not a predictive factor for local recurrence. When the $\mathrm{N}$ stage of the sixth TNM staging system was separately analyzed as a covariate, the LNR was also found to be a predictive factor for both OS and SRFS ( $\mathrm{P}=0.012$ and $\mathrm{P}=0.004$, respectively). A LNR value of 0.21 offered the best cut off to separate patients into two prognostic groups.

Conclusion: The defined cut-off values of the LNR were an independent risk factor for OS and distant metastasis-free survival in patients with rectal cancer, irrespective of the sixth or the seventh version of the TNM classification, and the LNR should be considered as a prognostic variable in any future staging system.

Keywords: Rectal cancer; Lymph node; Prognosis

\section{INTRODUCTION}

The prognosis of rectal cancer is still poor, with a survival rate of around $60 \%$ and a local recurrence rate of approximately $10 \%[1-3]$; therefore, many efforts to identify prognostic factors have continuously been made to improve the prognosis. Although one of the most critical prognostic factors is the status of lymph node metastasis $[4,5]$, the survival rate of stage III rectal cancer at the same stage varies $[4,6]$, showing its limitations in reflecting the prognosis. In addition, the existing $\mathrm{LN}$ staging of rectal cancer ( $\mathrm{N}$ staging) is based on only the number of metastatic lymph nodes $[4,6]$

Received: April 27, 2011 Accepted: August 30, 2011

Correspondence to: Kwan Hee Hong, M.D.

Department of Surgery, Busan Paik Hospital, Inje University College of

Medicine, Gaegeum-dong, Jin-gu, Busan 633-165, Korea

Tel: +82-51-890-6349, Fax: +82-51-898-9427

E-mail:gskhh@inje.ac.kr

(c) 2011 The Korean Society of Coloproctology

This is an open-access article distributed under the terms of the Creative Commons Attribution NonCommercial License (http://creativecommons.org/licenses/by-nc/3.0) which permits unrestricted non-

commercial use, distribution, and reproduction in any medium, provided the original work is properly cited. and does not reflect the number of investigated lymph nodes, to which a correlation with survival of colorectal cancer has been reported $[7,8]$.

The metastatic LN ratio (LNR), which can be classified with one to three cut-off values, is a significant prognostic factor for survival in stage III colon cancer, and its role as prognosticator has been reported to more valuable than that of the existing lymph node staging [9-11]. This prognostic value of the LNR in colon cancer is also reported in rectal cancer as a risk factor for mortality and recurrence $[12,13]$. A multicenter study reported a significant correlation between the LNR and survival in rectal cancer [14].

However, previous studies on the LNR in rectal cancer selected cut-off points arbitrarily [12] or included only patients undergoing sphincter-preserving surgery [13] or patients with over 70\% pelvic sidewall lymph node dissection [14]; therefore, the bias could have been in the patient selection. In addition, the studies had a limitation of methodology in that the clinical value of the LNR was analyzed by comparing $\mathrm{N}$ staging divided into two groups with the LNR divided into four groups $[12,14]$. For that reason, we divided stage III rectal cancer patients by using cut-off points of the LNR determined through statistical methods and analyzed the 
prognostic factors by using the TNM staging in the sixth [15] and the seventh [6] editions, respectively, to investigate the clinical value of the LNR in rectal cancer with lymph node metastasis.

\section{METHODS}

\section{Patients}

This study recruited 299 patients undergoing a proctectomy for rectal cancer within $10 \mathrm{~cm}$ from the anal verge measured with preoperatively rigid sigmoidoscopy or colonoscopy at Pusan Paik Hospital from January 1995 to December 2004. We excluded 100 patients with negative lymph node metastasis, distant metastasis found at diagnosis, history of preoperative adjuvant therapy and remaining local residual cancer left. We also excluded nine patients using postoperative adjuvant chemotherapy other than 5-fluorouracil (5-FL) or not being followed up, so 190 patients were finally included in this study. All surgery was performed by one surgeon, and it was open surgery based on a total mesorectal excision. An abdominoperineal resection was performed when the tumor distance was less than $4 \mathrm{~cm}$ from anal verge, invasion of the pelvic floor muscle caused by the tumor was suspected during the surgery or the distal resection margin was threatened by the tumor.

A combination of chemotherapy and radiotherapy was provided postoperatively, and its indication was patients with an involvement of the circumferential resection margin. However, it was optionally done when the number of metastatic lymph nodes was one to three in the postoperative pathological examination or when the patients were over 75 years or had heart disease. The postoperative adjuvant chemotherapy started within 2-4 weeks after the surgery and was given "Mayo regimen" for 5 days every month by bolus infusing 5-FL and leucovorin six times or by orally administering 5-FL for the elderly. Radiotherapy began on the first day of the third cycle of intravenous chemotherapy and at around four weeks after the surgery for the oral administration of chemotherapy. Fractionated irradiation of the whole pelvic cavity with a dose of 4,500-5,000 cGy was conducted. When the dose of intravenously administered chemotherapy and the dose of planned radiotherapy were accomplished fully, the patient was considered to have completed chemoradiation therapy.

After assessing the proximal resection margin, the distal resection margin, the integrity of the meorectal fascia, and the metastatic lymph nodes of the resected specimen through gross examination by the surgeon in the operating room, specimen was sent to pathology. The samples were fixed in $10 \%$ formalin solution for 24 hours, and lymph nodes were collected by dissecting them through manual palpation to examine lymph nodes of the mesorectum around the primary lesion and the proximal mesorectum. Special techniques, such as the fat-clearing method, were not used in the dissection of lymph nodes. Tissues showing firm texture under digital compression during the collection of lymph nodes were thought to be lymph nodes; lymph nodes with sizes of more than $3 \mathrm{~mm}$ were sectioned along the longitudinal axis, and those with sizes of less than $3 \mathrm{~mm}$ were used as a whole for hematoxilin-eosine staining and paraffin section manufacturing.

\section{Lymph node staging}

Lymph node stages were classified into N1 and N2 based on the sixth edition of the classification of the American Joint Committee on Cancer (AJCC) by using the number of metastatic lymph nodes and into N1a, N1b, N2a and N2b based on its seventh edition. To investigate the prognosis by the LNR of the number of metastatic lymph nodes to the number of collected and examined lymph nodes, its cut-off values were determined to be $0.21,0.32$, and 0.61 through recursive partitioning [16] using R 2.2.1 (R Development Core Team, Vienna, Austria). The patients were classified into four groups with the three cut-off values: group 1 (LNR1, $\mathrm{n}=74$ ) for LNRs of less than 0.21; group $2(\mathrm{LNR} 2, \mathrm{n}=25)$ for LNRs of 0.21-0.32; group 3 (LNR3, $\mathrm{n}=56$ ) for LNRs of 0.32-0.61; and group $4(\mathrm{LNR} 4, \mathrm{n}=35)$ for LNRs of over 0.61 .

\section{Follow-up and survival analysis}

Postoperative follow-up included physical examination and blood tests such as serum carcinoembryonic antigen every three months for first 2 years and every six months, after 2 years respectively. Radiologic examination, like abdominopelvic computerized tomography (CT), chest X-ray and chest CT, was performed every six or twelve months for the first two years and then every 12 months. If any suspicious lesion was found on radiologic examination, a bone scan and positron emission tomography were additionally performed.

Local recurrence was defined as a recurrence newly found in the pelvic cavity or the perineum in the postoperative follow-up and confirmed by biopsy or by a continuous increase of the size in the radiologic examinations conducted at intervals of 3-6 months. Systemic recurrence of the disease was defined pathologically or on the basis of radiologic findings during follow-up. If the serum carcinoembryonic antigen level became higher but there was no abnormality on radiologic examination, the patient was not considered as having a recurrence. The period to recurrence or of survival was calculated from the date of surgery for rectal cancer to the date of the last visit to the outpatient clinic or the date of death. If recurrence was not observed by the last follow-up, regardless of death, the case was considered as no recurrence. Deaths due to causes other than rectal cancer were considered as deaths in the survival analysis.

\section{Statistical analysis}

Statistical analysis was performed with SPSS ver. 12.0 (SPSS Inc., Chicago, IL, USA). The means of the groups were compared with one-way ANOVA, and chi-squared test and Fisher's exact tests were used to compare the frequencies of the four LNR groups with the clinicopathological variables. The Kaplan-Meier method was used to estimate the rates of survival and recurrence. Log rank tests were used to compare patient groups with respect to the estimated survival and recurrence curves. For independent prognostic factors, 
a multivariate analysis using the Cox regression model was used to determine factors influencing recurrence and death. Factors with a P-value of less than 0.1 in the univariate analysis were included in the multivariate analysis. A P-value of less than 0.05 was considered to be statistically significant. By considering that $\mathrm{N}$ stages of TNM staging, two groups in the sixth edition and four groups in the seventh edition, the LNRs were also classified into two and four groups, respectively, and the two groups and the four groups

Table 1. Characteristics of the patients in this study

\begin{tabular}{|c|c|}
\hline Variables & \\
\hline Age (yr) & $57.0(29-81)$ \\
\hline Gender (M:F) & $1.04: 1$ \\
\hline Follow-up duration (mo) & $55.0(6-145)$ \\
\hline \multicolumn{2}{|l|}{ Tumor location from anal verge (cm) } \\
\hline $0-5$ & $66(34.7)$ \\
\hline $5.1-10$ & $124(65.3)$ \\
\hline \multicolumn{2}{|l|}{ Operative method } \\
\hline Abdominoperineal resection & $77(40.5)$ \\
\hline Sphincter-saving operation & $113(59.5)$ \\
\hline \multicolumn{2}{|l|}{ T stage } \\
\hline $\mathrm{T} 1,2$ & $15(7.8)$ \\
\hline T3, 4 & $175(92.2)$ \\
\hline \multicolumn{2}{|l|}{ N stage } \\
\hline N1 & $107(56.3)$ \\
\hline N2 & $83(43.7)$ \\
\hline $\mathrm{N} 1 \mathrm{a}^{\mathrm{a}}$ & $56(29.5)$ \\
\hline$N 1 b^{a}$ & $51(26.8)$ \\
\hline $\mathrm{N} 2 \mathrm{a}^{\mathrm{a}}$ & $42(22.1)$ \\
\hline $\mathrm{N} 2 \mathrm{~b}^{\mathrm{a}}$ & $41(21.6)$ \\
\hline \multicolumn{2}{|l|}{ Lymphatic duct invasion } \\
\hline Present & $61(32.1)$ \\
\hline Absent & $129(67.9)$ \\
\hline \multicolumn{2}{|l|}{ Extramural venous invasion } \\
\hline Present & $27(14.2)$ \\
\hline Absent & $163(85.8)$ \\
\hline Carcinoembryonic antigen $(\mathrm{ng} / \mathrm{mL})>5^{\mathrm{b}}$ & $56(31.2)$ \\
\hline \multicolumn{2}{|l|}{ Tumor grade } \\
\hline High & $16(8.4)$ \\
\hline Low or medium & $174(91.6)$ \\
\hline Chemoradiation & $114(60.0)$ \\
\hline \multicolumn{2}{|l|}{ LN resected } \\
\hline No. of lymph node examined & $12(2-52)$ \\
\hline No. of lymph node positive & $3(1-32)$ \\
\hline
\end{tabular}

Values are presented as median (range) or number (\%).

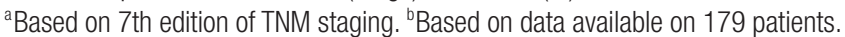

of the $\mathrm{N}$ staging and the LNR were compared in the multivariate analysis for a more objective statistical analysis.

\section{RESULTS}

\section{Comparison of the characteristics of the subjects and variables}

The median age of the patients was 57.0 years (range, 29 to 81 years) and the median follow-up period was 55 months (range, 6 to 145 months). Eighteen (9.4\%) patients were followed up for less than five years. The median number of lymph nodes retrieved was 12 (range, 2 to 52), and the median number of metastatic lymph nodes was 3 (range, 1 to 32 ) (Table 1). The number of the collected lymph nodes was inversely correlated to the LNR $(r=-0.085)$, but the correlation was not statistically significant $(\mathrm{P}=0.245)$ (Fig. 1). Although the four groups divided through recursive partitioning did not show any significant differences in comparison of rates with various clinicopathological variables, LNR4 had higher rates of N2 and $\mathrm{N} 2 \mathrm{~b}(\mathrm{P}=0.000)$, and LNR1 had relatively higher rates of $\mathrm{N} 1$ and N1a, respectively, in the sixth and the seventh editions $(\mathrm{P}=$ 0.000). Histologic differentiation of LNR3 and LNR4 showed significantly lower grades compared to LNR1 and LNR2 $(\mathrm{P}=0.009)$, and the proportions of patients with postoperative adjuvant therapy were not different among the groups divided by LNR (P = 0.361) (Table 2).

\section{Oncologic outcomes}

Thirty-four (18.5\%) patients had a local recurrence, and 78 (41.0\%) developed systemic recurrence during the follow-up period. Sixteen patients developed both local and systemic recurrence, and 90 (42.3\%) died during follow-up. In the univariate analysis, N staging in the sixth and the seventh editions, LNR and preoperative carcinoembryonic antigen level were significant factors of survival and systemic recurrence, and only $\mathrm{N}$ staging in the seventh edi-

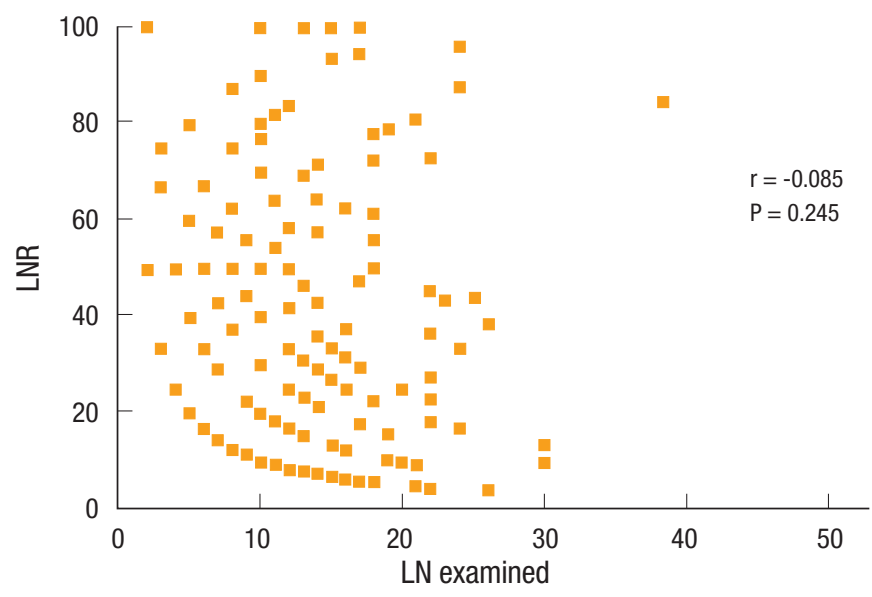

Fig. 1. Correlation between lymph node (LN) examined and lymph node ratio (LNR). 
Table 2. Comparison of the characteristics of patients by lymph node ratio (LNR)

\begin{tabular}{|c|c|c|c|c|c|}
\hline Variables & LNR1 $(n=74)$ & $\operatorname{LNR2}(n=25)$ & LNR3 $(n=56)$ & LNR4 $(n=35)$ & P-value \\
\hline Age (yr) & $59.0(34-81)$ & $60.0(31-80)$ & $56.0(35-73)$ & $54.0(29-79)$ & 0.160 \\
\hline Gender (M:F) & $35: 39$ & $15: 10$ & $28: 28$ & $19: 16$ & 0.708 \\
\hline Tumor location from anal verge $(\mathrm{cm})$ & & & & & 0.377 \\
\hline $0-5$ & $26(35.1)$ & $11(44.0)$ & $16(23.6)$ & $13(37.1)$ & \\
\hline $5.1-10$ & $48(64.9)$ & $14(56.0)$ & $40(70.4)$ & $22(62.9)$ & \\
\hline Operative method & & & & & 0.359 \\
\hline Abdominoperineal resection & $30(40.5)$ & $12(48.0)$ & $16(32.1)$ & $17(48.6)$ & \\
\hline Sphincter-saving operation & $44(59.5)$ & $13(52.0)$ & $40(67.9)$ & $18(51.4)$ & \\
\hline T stage & & & & & 0.375 \\
\hline $\mathrm{T} 1,2$ & $7(9.5)$ & $2(8.0)$ & $4(7.1)$ & $2(5.7)$ & \\
\hline T 3,4 & $67(90.5)$ & $23(92.0)$ & $52(92.9)$ & $33(94.3)$ & \\
\hline \multicolumn{6}{|l|}{$\mathrm{N}$ stage } \\
\hline $\mathrm{N} 1$ & $71(95.9)$ & $14(56.0)$ & $19(35.7)$ & $3(8.6)$ & 0.000 \\
\hline N2 & $3(4.1)$ & $11(44.0)$ & $37(64.3)$ & $32(91.4)$ & \\
\hline $\mathrm{N} 1 \mathrm{a}^{\mathrm{a}}$ & $49(69.1)$ & $4(16.0)$ & $3(15.8)$ & $0(0.0)$ & 0.000 \\
\hline $\mathrm{N} 1 \mathrm{~b}^{\mathrm{a}}$ & $22(26.8)$ & $10(40.0)$ & $16(28.6)$ & $3(8.6)$ & \\
\hline $\mathrm{N} 2 \mathrm{a}^{\mathrm{a}}$ & $3(4.1)$ & $11(44.0)$ & $23(46.8)$ & $5(14.3)$ & \\
\hline $\mathrm{N} 2 \mathrm{~b}^{\mathrm{a}}$ & $0(0.0)$ & $0(0.0)$ & $14(25.0)$ & $27(77.1)$ & \\
\hline Lymphatic duct invasion & & & & & 0.080 \\
\hline Present & $21(23.4)$ & $4(16.0)$ & $20(35.7)$ & $16(45.7)$ & \\
\hline Absent & $51(76.6)$ & $21(84.0)$ & $36(64.3)$ & $19(54.3)$ & \\
\hline Extramural venous invasion & & & & & 0.238 \\
\hline Present & $3(4.1)$ & $4(16.0)$ & $8(14.3)$ & $7(20.0)$ & \\
\hline Absent & $71(95.9)$ & $21(84.0)$ & $48(85.7)$ & $28(80.0)$ & \\
\hline Carcinormbryonic antigen $(\mathrm{ng} / \mathrm{mL})>5$ & $25(61.4)$ & $16(54.3)$ & $16(63.8)$ & $11(45.5)$ & 0.314 \\
\hline Tumor grade & & & & & 0.009 \\
\hline High & $3(4.1)$ & $1(4.0)$ & $5(8.9)$ & $7(20.0)$ & \\
\hline Low or medium & $71(95.9)$ & $24(96.0)$ & $51(91.1)$ & $28(80.0)$ & \\
\hline Chemoradiation & $50(67.6)$ & $15(60.0)$ & 30 (53.6) & 19 (55.3) & 0.351 \\
\hline
\end{tabular}

Data are presented as median (range) or number (\%) unless otherwise indicated.

a Based on 7th edition of TNM staging.

tion and LNR classified into two groups were significant factors of local recurrence. $\mathrm{N}$ staging in the sixth edition, LNR divided into four groups and the preoperative carcinoembryonic antigen level did not affect local recurrence significantly (Table 3). The five-year survival rate and the five-year systemic recurrence rate of LNR4 were $31.3 \%$ and $61.0 \%$, respectively, compared to those of LNR1, $72.0 \%$ and $26.0 \%$, respectively $(\mathrm{P}=0.001, \mathrm{P}=0.016)$. In the multivariate analysis, the risks of death and systemic recurrence were 3.9 times (95\% confidence interval [CI], 2.16 to 7.16 ) and 2.9 times (95\% CI, 1.58 to 3.33) higher in LNR4 than in LNR1, respectively, and the risk factors for death and systemic recurrence were found to be the preoperative carcinoembryonic antigen level and the LNR classified into two groups or into four groups. Neither $\mathrm{N}$ staging based on the sixth nor on the seventh edition were independent risk factors (Table 4). Univariate analysis revealed that there was interaction between LNR and retrieval of less than 12 lymph nodes in relation to survival and to systemic recurrence (Figs. 2, 3).

\section{DISCUSSION}

Lymph node metastasis is well known to be one of the most important prognostic factors in colorectal cancer. However, as the oncologic significance of the number of retrieved lymph nodes $[7,8]$ has increased, some have pointed out that the current $\mathrm{N}$ staging, 
Table 3. Univariate analysis of the risk factors

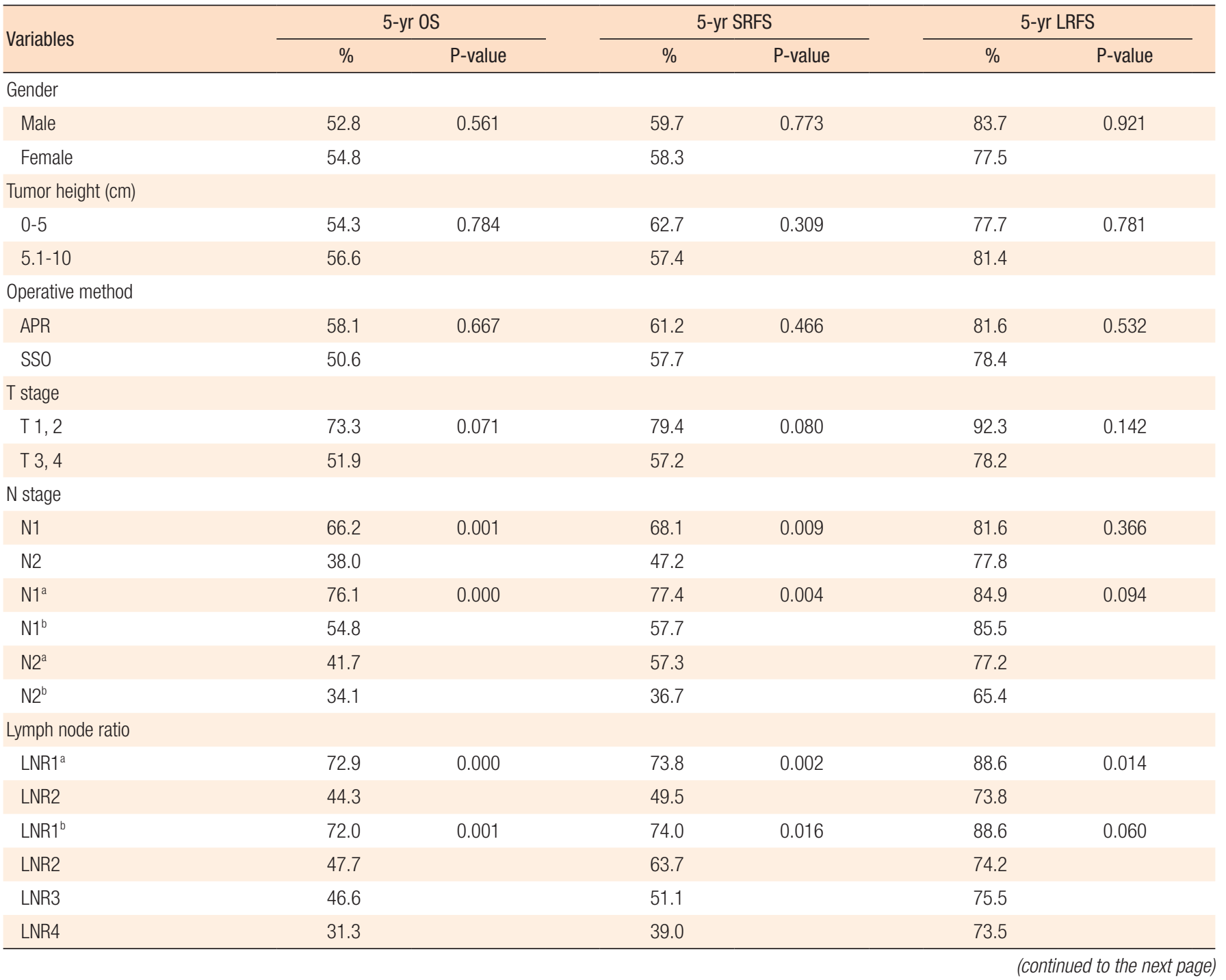

which depends on the number of metastatic lymph nodes $[4,6]$, cannot reflect the prognosis accurately. Furthermore, the number of retrieved lymph nodes is a variable affected by numerous factors [17], so a prognostic classification system to minimize the influence of the number of collected lymph nodes and to supplement the limitations of $\mathrm{N}$ staging is necessary for postoperative adjuvant therapy and close follow-up in stage III colorectal cancer patients. In addition, because the survival rate of stage III colorectal cancer varies widely $[4,6,18]$, the clinical role of the LNR as a biologic predictor of prognosis has been demonstrated [9-14].

Previous studies on the LNR for rectal cancer $[12,14,19]$ determined cut-off values based on quartiles or complex statistical methods used, so the results had limitations in applications to clinical practice. Thus, we identified the cut-off values for the prognostic stratification by using recursive partitioning [20], which could sta- tistically calculate the cut-off values for the stratification of the prognostic groups in a relatively simple manner. In addition, previous studies on colorectal cancer [9-14] reporting the prognostic superiority of the LNR compared to current $\mathrm{N}$ staging have limitations of bias in the statistical analysis and the methodology. Therefore, we classified the LNR into two groups and four groups, respectively, and $\mathrm{N}$ staging into the two and the four groups, respectively, and used them as covariates, as categorical variables in multivariate analysis, to investigate whether the LNR or N staging was more effective in reflecting the prognosis. In this study, the LNR was found to be an independent factor increasing risk of death and systemic recurrence regardless of the method of $\mathrm{N}$ substaging, which means that the prognostic stratification of the LNR was superior to that of $\mathrm{N}$ staging. Our results were similar to those in a report by De Ridder et al. [11] demonstrating that the LNR had better ability to 
Table 3. (continued from the previous page) Univariate analysis of the risk factors

\begin{tabular}{|c|c|c|c|c|c|c|}
\hline \multirow{2}{*}{ Variables } & \multicolumn{2}{|c|}{5 -yr OS } & \multicolumn{2}{|c|}{ 5-yr SRFS } & \multicolumn{2}{|c|}{ 5-yr LRFS } \\
\hline & $\%$ & P-value & $\%$ & P-value & $\%$ & P-value \\
\hline \multicolumn{7}{|l|}{ No. of LN examined } \\
\hline$>12$ & 53.8 & 0.722 & 61.0 & 0.538 & 77.3 & 0.370 \\
\hline$<12$ & 53.6 & & 56.6 & & 82.2 & \\
\hline \multicolumn{7}{|l|}{$\mathrm{CRT}^{\mathrm{C}}$} \\
\hline Complete & 56.7 & 0.348 & 61.5 & 0.981 & 81.6 & 0.748 \\
\hline Incomplete & 51.1 & & 57.1 & & 78.2 & \\
\hline \multicolumn{7}{|l|}{ CEA $(n g / m L)^{d}$} \\
\hline$\leq 5$ & 66.9 & 0.000 & 69.1 & 0.000 & 82.8 & 0.064 \\
\hline$>5$ & 30.2 & & 40.8 & & 74.1 & \\
\hline \multicolumn{7}{|c|}{ Lymphatic duct invasion } \\
\hline Present & 57.3 & 0.719 & 62.2 & 0.481 & 74.0 & 0.435 \\
\hline Absent & 52.2 & & 57.7 & & 82.6 & \\
\hline \multicolumn{7}{|c|}{ Extramural venous invasion } \\
\hline Present & 44.1 & 0.295 & 61.0 & 0.401 & 78.7 & 0.377 \\
\hline Absent & 55.9 & & 47.0 & & 85.0 & \\
\hline \multicolumn{7}{|c|}{ Distal resection margin $(\mathrm{cm})^{e}$} \\
\hline$>1$ & 52.5 & 0.667 & 60.7 & 0.822 & 80.8 & 0.181 \\
\hline$<1$ & 53.5 & & 57.2 & & 82.9 & \\
\hline Close $^{f}$ & 34.1 & & 37.4 & & 56.1 & \\
\hline \multicolumn{7}{|l|}{ Tumor grade } \\
\hline High & 55.8 & 0.295 & 54.1 & 0.445 & 87.5 & 0.741 \\
\hline Low or medium & 49.2 & & 59.6 & & 78.9 & \\
\hline
\end{tabular}

OS, overall survival; SRFS, systemic recurrence-free survival; LRFS, local recurrence-free survival; APR, abdominoperineal resection; SSO, sphincter-saving operation including Hartmann's procedure; LNR, lymph node ratio; LN, lymph node; CRT, chemoradiation; CEA, carcinoembryonic antigen.

Cut-off values were ${ }^{\mathrm{a}} 0.21$ and ${ }^{\mathrm{b}} 0.21,0.32$, and 0.61 . ${ }^{\circ}$ Completeness of CRT was defined as intended doses of radiation and chemotherapy given, ${ }^{\mathrm{d}} 11$ patients in whom serum CEA level was not measured were excluded from the analysis. ${ }^{e}$ Only the patients with SSO were included in the analysis. ${ }^{\mathrm{f}}$ The distal clearance margin of $2 \mathrm{~mm}$ or less whereas the margin was microscopically uninvolved.

Table 4. Multivariate analysis of survival and systemic recurrence-free survival

\begin{tabular}{|c|c|c|c|c|c|}
\hline \multirow{2}{*}{ Variables } & \multirow{2}{*}{ Category } & \multicolumn{2}{|c|}{ Overall survival } & \multicolumn{2}{|c|}{ Systemic recurrence-free survival } \\
\hline & & Adjusted HR $(95 \% \mathrm{Cl})$ & P-value & Adjusted HR $(95 \% \mathrm{Cl})$ & P-value \\
\hline CEA level $(\mathrm{ng} / \mathrm{mL})$ & $\leq 5$ & 1 & 0.000 & 1 & 0.000 \\
\hline \multirow[t]{2}{*}{$\mathrm{LNR}^{\mathrm{a}}$} & 1 & 1 & 0.012 & 1 & 0.004 \\
\hline & 2 & $2.07(1.11-3.77)$ & & $2.20(1.28-3.56)$ & ' \\
\hline & 3 & $2.48(1.27-4.84)$ & & $2.20(1.19-3.20)$ & \\
\hline & 4 & $3.94(2.16-7.16)$ & & $2.94(1.58-3.33)$ & \\
\hline
\end{tabular}

HR, hazard ratio; $\mathrm{Cl}$, confidence interval; CEA, carcinoembryonic antigen; LNR, lymph node ratio.

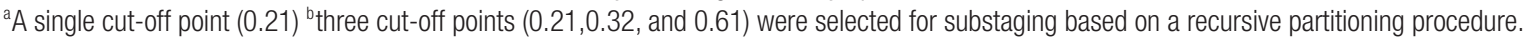




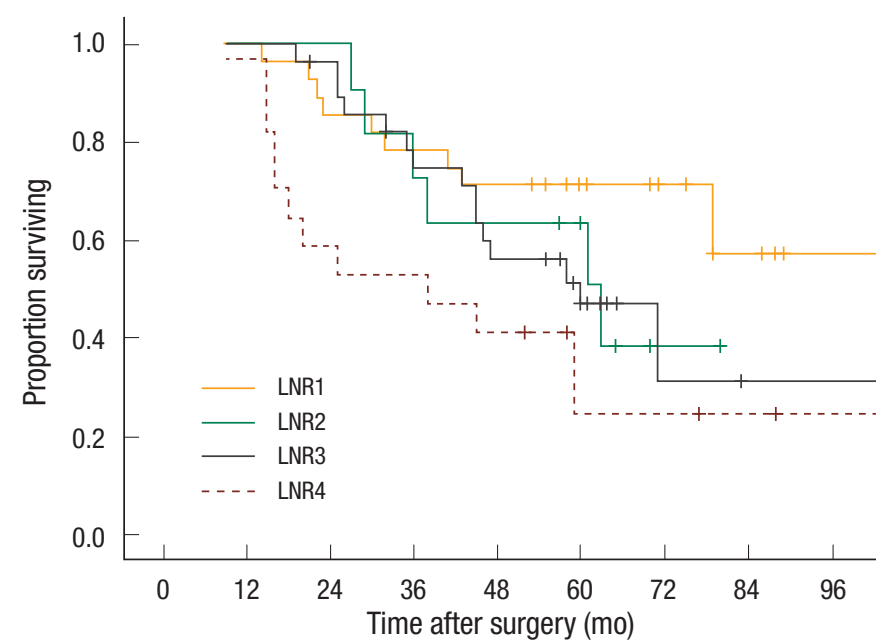

Fig. 2. Survival curve for subgroups according to lymph node ratio (LNR) in patients with inadequate numbers of examined $(\mathrm{P}=0.037)$.

discriminate prognosis groups by dividing the LNR and $\mathrm{N}$ stages into two groups, respectively, only in colon cancer.

Given the hypothesis of Rinkus et al. [21] that preoperative adjuvant therapy could raise the absolute value of the LNR by reducing the number of collected lymph nodes, it seems to rational to exclude patients from receiving neoadjuvant therapy to decrease the possibility of neoadjuvant therapy influencing the LNR. Moreover, a previous studies $[13,14]$ on the LNR in patients with low rectal cancer found that the LNR had an oncologic significance, but those results should be carefully interpreted due to limitations in patient selection. However, considering this study included the patients who were not treated with neoadjuvant treatment, our results might provide clinical data on treatment of stage III mid and low rectal cancer although its number of subjects is small and it utilizes a retrospective study design.

One of the major limitations in previous studies on the LNR was the subjective determination of cut-off values, which was pointed out by Ceelen et al. [22]. Thus, we used recursive partitioning, as mentioned above, to determine the cut-off values in a more objective and useful manner $[16,20]$ and selected a single reference point, 0.21 , for the prognostic classification. Dekker et al. [19] reporting the oncologic impact of the LNR in rectal cancer patients receiving neoadjuvant therapy identified a single cut-off value of 0.6 . The cut-off value of 0.6 according to Dekker et al. [19] is quite different from that of this study of 0.21 , and the difference can be partly explained by the assumption that preoperative adjuvant therapy can increase the absolute value of the LNR by decreasing the number of non-metastatic lymph nodes [21]. However, the statistically calculated cut-off values of $0.21,0.32$, and 0.61 in this study were within the range of the cut-off values in previous studies [12, 23], so the cut-off values of the LNR to stratify the prognosis were within a certain range in spite of the diversity of the methodology used to determine them.

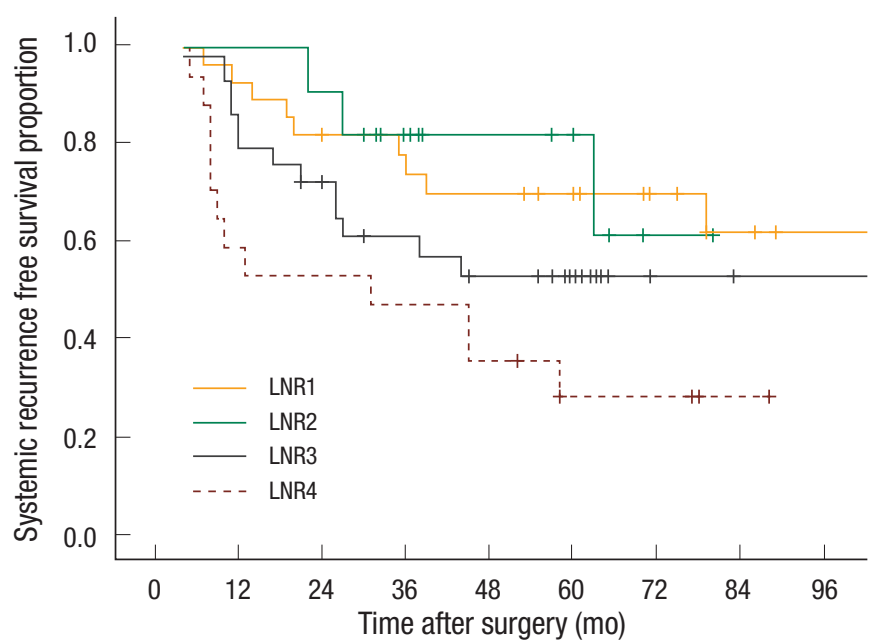

Fig. 3. Systemic recurrence free survival curve for subgroups according to lymph node ratio (LNR) in patients with inadequate numbers of nodes examined $(\mathrm{P}=0.019)$.

For the impact of the LNR on survival and systemic recurrence, the ratio was found to be a risk factor of death and systemic recurrence, unlike the correlation with local control, even after adjusting various other clinical variables, including $\mathrm{N}$ staging, and this finding confirmed the results of previous studies [12-14]. It shows that the LNR is an important prognostic factor for death or recurrence and suggests the need for clinical efforts to reduce death and systemic recurrence in patients with a high LNR regardless of the current $\mathrm{N}$ staging. Moreover, the multivariate analysis of survival and systemic recurrence did not find a prognostic value of $\mathrm{N}$ staging in this study, which is consistent with the result of Ceelen et al. [22] that the LNR is a better prognostic factor than the number of metastatic lymph nodes in colorectal cancer and which is similar with those of Kim et al. [12] and Dekker et al. [19]. These results imply limitations of the existing $\mathrm{N}$ staging, which depends on only the number of metastatic lymph nodes, and suggest the need for a prognostic classification system based on LNR, refreflecting the numbers of collected lymph nodes.

Some reports demonstrated that the clinical significance of the LNR was not related with the numbers of the collected lymph node in colorectal cancer [12, 24], and others showed contrary results $[10,25]$. According to a study of Kim et al. [12] on the LNR in rectal cancer, the ratio had an oncologic impact regardless of the number of retrieved lymph nodes with a cutoff value of 17 , and in the study of Rosenberg et al. [24], the correlation between LNR and survival in patients with a number of retrieved lymph nodes of less than 12 implied that the LNR was a clinical variable not much affected by the number of collected lymph nodes. In this study, because the cases with a number of collected lymph nodes of less than 12 was small we only performed a univariate analysis. However, the survival rate was significantly different by LNR, and this finding supports that of Rosenberg et al. [24].

The LNR divided into ranges based on cutoff values calculated 
through recursive partitioning showed better prognostic predictability than the current lymph node staging system in terms of death of and systemic recurrence in rectal cancer patients, and the oncologic significance of the LNR classification was observed even when the number of collected lymph nodes was less than 12 . Therefore, the LNR needs to be considered in any future staging system.

\section{CONFLICT OF INTEREST}

No potential conflict of interest relevant to this article was reported.

\section{REFERENCES}

1. Sauer R, Becker H, Hohenberger W, Rodel C, Wittekind C, Fietkau $\mathrm{R}$, et al. Preoperative versus postoperative chemoradiotherapy for rectal cancer. N Engl J Med 2004;351:1731-40.

2. Kapiteijn E, Marijnen CA, Nagtegaal ID, Putter H, Steup WH, Wiggers T, et al. Preoperative radiotherapy combined with total mesorectal excision for resectable rectal cancer. N Engl J Med 2001;345:638-46.

3. Zaheer S, Pemberton JH, Farouk R, Dozois RR, Wolff BG, Ilstrup D. Surgical treatment of adenocarcinoma of the rectum. Ann Surg 1998;227:800-11.

4. Greene FL, Stewart AK, Norton HJ. New tumor-node-metastasis staging strategy for node-positive (stage III) rectal cancer: an analysis. J Clin Oncol 2004;22:1778-84.

5. den Dulk M, Marijnen CA, Putter H, Rutten HJ, Beets GL, Wiggers $\mathrm{T}$, et al. Risk factors for adverse outcome in patients with rectal cancer treated with an abdominoperineal resection in the total mesorectal excision trial. Ann Surg 2007;246:83-90.

6. Gunderson LL, Jessup JM, Sargent DJ, Greene FL, Stewart A. Revised tumor and node categorization for rectal cancer based on surveillance, epidemiology, and end results and rectal pooled analysis outcomes. J Clin Oncol 2010;28:256-63.

7. Baxter NN, Virnig DJ, Rothenberger DA, Morris AM, Jessurun J, Virnig BA. Lymph node evaluation in colorectal cancer patients: a population-based study. J Natl Cancer Inst 2005;97:219-25.

8. Goldstein NS. Lymph node recoveries from 2427 pT3 colorectal resection specimens spanning 45 years: recommendations for a minimum number of recovered lymph nodes based on predictive probabilities. Am J Surg Pathol 2002;26:179-89.

9. Lee HY, Choi HJ, Park KJ, Shin JS, Kwon HC, Roh MS, et al. Prognostic significance of metastatic lymph node ratio in node-positive colon carcinoma. Ann Surg Oncol 2007;14:1712-7.

10. Berger AC, Sigurdson ER, LeVoyer T, Hanlon A, Mayer RJ, Macdonald JS, et al. Colon cancer survival is associated with decreasing ratio of metastatic to examined lymph nodes. J Clin Oncol 2005;23:8706-12.

11. De Ridder M, Vinh-Hung V, Van Nieuwenhove Y, Hoorens A, Sermeus A, Storme G. Prognostic value of the lymph node ratio in node positive colon cancer. Gut 2006;55:1681.
12. Kim YS, Kim JH, Yoon SM, Choi EK, Ahn SD, Lee SW, et al. lymph node ratio as a prognostic factor in patients with stage III rectal cancer treated with total mesorectal excision followed by chemoradiotherapy. Int J Radiat Oncol Biol Phys 2009;74:796-802.

13. Peng J, Xu Y, Guan Z, Zhu J, Wang M, Cai G, et al. Prognostic significance of the metastatic lymph node ratio in node-positive rectal cancer. Ann Surg Oncol 2008;15:3118-23.

14. Kobayashi H, Mochizuki H, Kato T, Mori T, Kameoka S, Shirouzu $\mathrm{K}$, et al. Lymph node ratio is a powerful prognostic index in patients with stage III distal rectal cancer: a Japanese multicenter study. Int J Colorectal Dis 2011;26:891-6.

15. Puppa G, Sonzogni A, Colombari R, Pelosi G. TNM staging system of colorectal carcinoma: a critical appraisal of challenging issues. Arch Pathol Lab Med 2010;134:837-52.

16. Breiman L, Friedman J, Stone CJ, Olshen RA. Classification and Regression Trees. Belmont, CA: Wadsworth International Group; 1984.

17. Mekenkamp LJ, van Krieken JH, Marijnen CA, van de Velde CJ, Nagtegaal ID; Pathology Review Committee and the Co-operative Clinical Investigators. Lymph node retrieval in rectal cancer is dependent on many factors: the role of the tumor, the patient, the surgeon, the radiotherapist, and the pathologist. Am J Surg Pathol 2009;33:1547-53.

18. Gunderson LL, Jessup JM, Sargent DJ, Greene FL, Stewart AK. Revised TN categorization for colon cancer based on national survival outcomes data. J Clin Oncol 2010;28:264-71.

19. Dekker JW, Peeters KC, Putter H, Vahrmeijer AL, van de Velde CJ. Metastatic lymph node ratio in stage III rectal cancer; prognostic significance in addition to the 7th edition of the TNM classification. Eur J Surg Oncol 2010;36:1180-6.

20. Cook EF, Goldman L. Empiric comparison of multivariate analytic techniques: advantages and disadvantages of recursive partitioning analysis. J Chronic Dis 1984;37:721-31.

21. Rinkus KM, Russell GB, Levine EA. Prognostic significance of nodal disease following preoperative radiation for rectal adenocarcinoma. Am Surg 2002;68:482-7.

22. Ceelen W, Van Nieuwenhove Y, Pattyn P. Prognostic value of the lymph node ratio in stage III colorectal cancer: a systematic review. Ann Surg Oncol 2010;17:2847-55.

23. Moug SJ, McColl G, Lloyd SM, Wilson G, Saldanha JD, Diament RH. Comparison of positive lymph node ratio with an inflammation-based prognostic score in colorectal cancer. Br J Surg 2011; 98:282-6.

24. Rosenberg R, Friederichs J, Schuster T, Gertler R, Maak M, Becker $\mathrm{K}$, et al. Prognosis of patients with colorectal cancer is associated with lymph node ratio: a single-center analysis of 3,026 patients over a 25-year time period. Ann Surg 2008;248:968-78.

25. Park IJ, Choi GS, Jun SH. Nodal stage of stage III colon cancer: the impact of metastatic lymph node ratio. J Surg Oncol 2009;100: 240-3. 